\title{
Ueber die linearen Differentialgleichungen, welchen die Periodicitätsmoduln der Abelschen Integrale genügen, und über verschiedene Arten von Differentialgleichungen für $\vartheta(0,0, \ldots 0)$.
}

\author{
(Von Herrn L. Fuchs in Greifswald.)
}

In einer früheren Abhandlung (d. J. B. 71) haben wir die Periodicitätsmoduln der hyperelliptischen Integrale als Functionen eines Parameters einer näheren Untersuchung unterzogen und namentlich auch die linearen Differentialgleichungen aufgestellt, welchen diese Functionen genügen. In dem Folgenden wollen wir ebenso für die Periodicitätsmoduln der allgemeinen Abelschen Integrale, als Functionen eines der sich nicht aufhebenden Verzweigungspunkte der der Theorie zu Grunde gelegten Fläche T, lineare Differentialgleichungen entwickeln, unter der Voraussetzung, dass die sich nicht aufhebenden Verzweigungspunkte von einander unabhängig sind.

In diesem Journale B. 36 hat Jacobi für das elliptische $\boldsymbol{\vartheta}(0)$ als Function von $\log q$ eine Differentialgleichung dritter Ordnung entwickelt, deren Coefficienten numerische Grössen sind. Dieselbe stẹt in enger Beziehung zu der bekannten linearen Differentialgleichung zweiter Ordnung, welcher $K$ und $K^{\prime}$ genügen, und sie lässt eine unmittelbare Anwendung auf die Transformation zu. - Wir wollen im Folgenden zeigen, dass ähnliche Relationen sich in der Theorie der Abelschen Functionen zwischen $\boldsymbol{\vartheta}(0,0, \ldots 0)$ als Function der Periodicitätsmoduln und deren partiellen Ableitungen nach denselben ergeben, und dass die Herleitung derselben entweder mit Hülfe der bei den oben angeführten Differentialgleichungen der Periodicitätsmoduln in dieser Arbeit hergestellten Beziehungen, oder auch unabhängig von diesen möglich ist.

Da jedoch die Periodicitätsmoduln nicht von einander unabhängig sind, so scheinen uns für manche andere Zwecke, als die sich unmittelbar auf das Problem der Transformation beziehenden, solche Differentialgleichungen den Vorzug zu verdienen, welchen $\boldsymbol{9}(0,0, \ldots 0)$ als Function eines der $3 p-3$ Klassenmoduln, oder eines der als unabhängig von einander vorausgesetzten sich.nicht aufhebenden Verzweigungspunkte genügen. Wir wählen die letzteren 
als unabhängige Variabeln und zeigen, dass $\vartheta(0,0, \ldots 0)$ als Function derselben gewisse algebraische Differentialgleichungen befriedigt.

In der Bezeichnung schliessen wir uns überall an die Abhandlung von Riemann (d. J. B. 54) an, welche wir kurz als R. A. F. citiren.

\section{1.}

Ehe wir in unsere Untersuchung eintreten, ist es nöthig nachzuweisen, dass die sich nicht aufhebenden Verzweigungspunkte der die algebraische Gleichung

$$
\text { (1.) } \quad F(\stackrel{n}{s}, \boldsymbol{z})=0
$$

darstellenden Fläche $T$ als von einander unabhängig vorausgesetzt werden dürfen.

Zwischen der Anzahl $w$ dieser Punkte, und der Anzahl $2 p$ von Querschnitten, welche die Fläche $T$ in eine einfach zusammenhangende verwandeln, findet die Beziehung statt:

$$
\text { (2.) } w=2 n+2(p-1) \quad \text { (R. A. F. \$.7). }
$$

Für die mit Gleichung (1.) zu derselben Klasse gehörigen algebraischen Gleichungen ist $p$ unveränderlich (R. A. F. S. 11). Die Gleichung niedrigsten Grades in derselben Klasse hat für $p>2$ die Form

und es ist

$$
\text { (3.) } \quad F_{1}(s, z)=0
$$

$$
\text { (4.) } p=2 \nu-\varepsilon \text {, }
$$

wo $\varepsilon=2$ oder 3 , je nachdem $p$ gerade oder ungerade. (R. A. F. \$. 13).

Es sei $w_{1}$ die Anzahl der sich nicht aufhebenden Verzweigungspunkte der Gleichung (3.), so ist nach Gleichung (2.)

$$
\text { (5.) } \quad w_{1}=2 \nu+2(p-1) \text {, }
$$

oder

$$
\text { (6.) } w_{1}=6 v-2(1+\varepsilon) \text {. }
$$

Die Anzahl $(\nu+1)^{2}$ der Constanten der Gl. (3.) ist also um mehr als eine Einheit grösser als $w_{1}$.

Nun sei

$$
\text { (7.) } n=\nu+\zeta, \quad m=\nu+\eta,
$$

wo $\zeta$ und $\eta$ entweder Null oder positive ganze Zahlen sind, es folgt alsdann aus Gl. (2.) und (5.)

(8.) $\quad \omega=w_{1}+2 \zeta$. 
326 Fuchs, üb. gewisse Differentialgleichungen in d. Theorie d. Abelschen Integrale.

Die Anzahl der Constanten der Gl. (1.) ist

$$
(m+1)(n+1)=(\nu+1+\zeta)(\nu+1+\eta)=(\nu+1)^{2}+(\nu+1+\eta) \zeta+(\nu+1) \eta,
$$

also ühertrifft die Anzahl der Constanten der Gl. (1.) die der Gleichung (3.) um mehr als $2 \zeta$, und demnach die Anzahl $w$ der sich nicht aufhebenden Verzweigungspunkte der Gleichung (1.) um mehr als eine Einheit, so dass im Allgemeinen letzteren Punkten willkürliche, von einander unabhängige Lagen gegeben werden können.

Zu demselben Schlusse gelangt man auch für $p=1$ oder 2 . Wir werden demnach in dieser Arbeit die sich nicht aufhebenden Verzweigungspunkte als von einander unabhängig betrachten.

2.

Eine fernere Bemerkung bezieht sich auf die Darstellung einer rationalen Function von $(s, z)$, die in gegebenen Punkten unendlich gross und in anderen gegebenen unendlich klein werden soll.

Bekanntlich kann man mit Hülfe der Gleichung

$$
\text { (1.) } \quad F(s, m)=0
$$

jede rationale Function $f(s, z)$ auf die Form bringen:

$$
f(s, z)=\frac{L_{n-1} s^{n-1}+L_{n-2} s^{n-2}+\cdots+L_{0}}{N},
$$

wo die Grössen $L_{n-1}, L_{n-2}, \ldots L_{0}$ und $N$ ganze rationale Functionen von z sind.

Soll daher $f(s, z)$ in den Punkten $\left(s_{1}, z_{1}\right),\left(s_{2}, z_{2}\right), \ldots\left(s_{m}, z_{m}\right)$ unendlich gross erster Ordnung, sonst aber überall endlich werden, und setzen wir

$$
N=\left(z-z_{1}\right)\left(z-z_{2}\right) \ldots\left(z-z_{m}\right)
$$

und bezeichnen die $n-1$ übrigen Punkte der Fläche $T$, welche mit $\left(s_{\mathfrak{a}}, z_{\mathfrak{a}}\right)$ zu demselben Werthe $z_{\mathfrak{a}}$ gehören, mit $\left(s_{\mathfrak{a}}^{\prime}, z_{\mathfrak{a}}\right),\left(s_{\mathfrak{a}}^{\prime \prime}, z_{\mathfrak{a}}\right), \ldots\left(s_{\mathfrak{a}}^{(n-1)}, z_{\mathfrak{a}}\right)$, so ist der Zähler so zu bestimmen, dass er in den Punktèn $\left(s_{\mathfrak{a}}^{\prime}, z_{\mathfrak{a}}\right),\left(s_{\mathfrak{a}}^{\prime \prime}, z_{\mathfrak{a}}\right), \ldots\left(s_{\mathfrak{a}}^{(n-1)}, z_{\mathfrak{a}}\right)$ $(\mathfrak{a}=1,2, \ldots m)$ von der ersten Ordnung verschwindet. Diese $m(n-1) \mathrm{Be}-$ dingungen liefern ebenso viele lineare Gleichungen für die Coefficienten der $L$ als Unbekannte. - Ist $\mu$ die Anzahl dieser Coefficienten und

$$
\mu-m(n-1)=\nu+1,
$$


so treten zu jenen Gleichungen noch $v$ andere hinzu, welche ausdrücken, dass der Zähler von $f(s, z)$ ausserdem in den $\nu$ gegebenen Punkten $\left(b_{1}, a_{1}\right)$, $\left(b_{2}, a_{2}\right), \ldots\left(b_{v}, a_{v}\right)$ erster Ordnung verschwinden soll. Die Gesammtheit der $\mu-1$ Gleichungen liefert die Verhältnisse der Coefficienten der $L$ als rationale Functionen der Grössen $\left(s_{\mathfrak{a}}^{\prime}, z_{\mathfrak{a}}\right),\left(s_{\mathfrak{a}}^{\prime \prime}, z_{\mathfrak{a}}\right), \ldots\left(s_{\mathfrak{a}}^{(n-1)}, z_{\mathfrak{a}}\right)$ und $\left(b_{1}, a_{1}\right),\left(b_{2}, a_{2}\right), \ldots\left(b_{v}, a_{v}\right)$. Da diese Functionen, wie sich aus der Theorie der linearen Gleichungen ergiebt, in Bezug auf $s_{\mathfrak{a}}^{\prime}, s_{\mathfrak{a}}^{\prime \prime}, \ldots s_{\mathfrak{a}}^{(n-1)}$ symmetrisch sind, und die symmetrischen Functionen dieser Grössen sich rational durch die Coefficienten der verschiedenen Potenzen der Gleichung

$$
\text { (1'.) } \quad \stackrel{n}{n}\left(s^{m}, z_{\mathfrak{a}}\right)=0
$$

und durch $s_{\mathfrak{a}}$ ausdrücken lassen, so ergiebt sich schliesslich der Satz:

Die Constanten in der rationalen Function $f(s, z)$, welche dadurch bestimmt wird, dass sie in den gegebenen Punkten $\left(s_{1}, z_{1}\right),\left(s_{2}, z_{2}\right), \ldots\left(s_{m}, z_{m}\right)$ unendlich gross erster Ordnung, sonst ïberall endlich, und in den gegebenen Punkten $\left(b_{1}, a_{1}\right),\left(b_{2}, a_{2}\right), \ldots\left(b_{v}, a_{v}\right)$ unendlich klein erster Ordnung wird, lassen sich als rationale Functionen dieser beiden Reihen von Grössenpaaren darstellen, deren Coefficienten ihrerseits rational aus den Constanten der Gleichung (1.) zusammengesetzt sind.

Wenn $m>p$, so ist nach (R. A. F. \$. 5 u. 8) die Bestimmung einer rationalen Function gemäss den obigen Bedingungen stets möglich, und es ergiebt sich $\nu=m-p$, so wie dass der Zähler derselben noch ausserdem in p Punkten verschwindet.

3.

Es sei

$$
\text { (1.) } \quad U=\int \frac{\varphi\left(\begin{array}{l}
n-2, m-2 \\
s, z
\end{array}\right)}{\frac{\partial F}{\partial s}} d z
$$

irgend ein Integral erster Gattung (R. A. F. \$.9), und $k$ irgend einer der sich nicht aufhebenden Verzweigungspunkte, so ist $\frac{\partial^{\lambda} U}{\partial k^{\lambda}}$ in der Fläche $T$ überall ausser in $k$ endlich und hat in der Umgebung von $k$ die Form

$$
\frac{\partial^{\lambda} U}{\partial k^{\lambda}}=c_{\lambda}(z-k)^{-\frac{2 \lambda-1}{2}}+c_{\lambda-1}(z-k)^{-\frac{2 \lambda-3}{2}}+\cdots+c_{1}(z-k)^{-\frac{k}{z}}+G,
$$

wo $G$ nur positive ganzzahlige Potenzen von $(z-k)^{\frac{1}{z}}$ enthält. 
Es seien nun $\varepsilon_{1}, \varepsilon_{2}, \ldots \varepsilon_{p} p$ willkürliche, aber feste Punkte der Fläche $T$, so lässt sich eine rationale Function $\chi_{\lambda}(s, z)$ derart bestimmen, dass der Nenner derselben in $k$ unentlich klein der Ordnung $2 \lambda-1$, in den Punkten $\varepsilon$ unendlich klein erster Ordnung wird, während ihr Zähler für die noch übrigen Nullpunkte des Nenners von gleicher Ordnung wie dieser verschwindet. Alsdann enthält der Zähler noch $2 \lambda$ willkürliche Constanten (R. A. F. \$. 8), über welche wir folgendermassen verfügen. In der Umgebung von $k$ hat $\chi_{\lambda}(s, z)$ die Form:

$$
\chi_{\lambda}(s, z)=(z-k)^{-\frac{2 \lambda-1}{2}}\left(e_{0}+e_{1}(z-k)^{\frac{1}{2}}+e_{2}(z-k)^{\frac{2}{2}}+\cdots+c_{2 \lambda-2}(z-k)^{\frac{2 \lambda-2}{2}}\right)+H,
$$

wo $H$ nur positive ganzzahlige Potenzen von $(z-k)^{\frac{1}{2}}$ enthält. Wir setzen nun

$$
\text { (4.) }\left\{\begin{array}{l}
e_{1}=e_{3}=\cdots=e_{2 \lambda-3}=0, \\
e_{1}=c_{\lambda}, \quad e_{2}=c_{\lambda-1}, \ldots e_{2 \lambda-2}=c_{1} .
\end{array}\right.
$$

Dieses giebt im Ganzen 2 $\lambda-1$ Bedingungen, gerade so viel, als zulässig sind.

Es ist alsdann

$$
\frac{\partial^{\lambda} U}{\partial k^{\lambda}}-\chi_{\lambda}(s, z)
$$

in den Punkten $\varepsilon$ unendlich gross erster Ordnung, sonst aber überall endlich. Sind daher $t_{1}, t_{2}, \ldots t_{p}$ Integrale zweiter Gattung von der Beschaffenheit, dass $t_{\nu}$ in $\varepsilon_{\nu}$ unendlich gross erster Ordnung, sonst überall endlich ist, sind ferner $U_{1}, U_{2}, \ldots U_{p}$ ein System linearunabhängiger Integrale erster Gattung, so dass

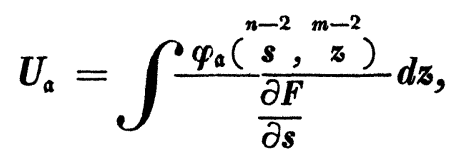

so ist (R. A. F. S. 5)

(5.) $\frac{\partial^{\lambda} U}{\partial k^{\lambda}}-\chi_{\lambda}=\delta_{\lambda 1} t_{1}+\delta_{\lambda 2} t_{2}+\cdots+\delta_{\lambda p} t_{p}+\gamma_{\lambda 1} U_{1}+\gamma_{\lambda 2} U_{2}+\cdots+\gamma_{\lambda p} U_{p}+$ Const., wo die Grössen $\gamma$ und $\delta$ Constanten bedeuten.

4.

Die Grössen $\gamma, \delta$ in Gl. (5.) vor. No. lassen sich, wenn die Function $\chi_{\lambda}$ und die Integrale $t_{\nu}$ gebildet sind, auf algebraischem Wege berechnen. Differentiiren wir daher die Gleichung (5.) vor.'No. nach $z$, und bringen die rationalen Functionen von $(s, z)$ auf beiden Seiten der erhaltenen Gleichung 
Fuchs, üb. gewisse Differentialgleichungen in d. Theorie d. Abelschen Integrale. 329

auf die Form der Gl. (2.) No. 2., so ergeben sich, wegen der vorausgesetzten Irreductibilität der Gl. (1.) No.1., $n$ Gleichungen, welche die Grössen $\gamma, \delta$ linear enthalten. Da diese Gleichungen identisch für jeden Werth von $z$ bestehen, so resultiren aus denselben lineare Gleichungen für die Grössen $\gamma, \delta$, welche für dieselben bestimmte Werthe liefern, da die Existenz der Gl. (5.) vor. No. bereits erwiesen. Sind $\left(\sigma_{v}, \zeta_{v}\right)$ die dem Punkte $\varepsilon_{v}$ zugehörigen Werthenpaare $(s, z)$, so wie $\sigma$ der dem $k$ zugehörige Werth von $s$, so folgt aus der Bildungsweise der rationalen Functionen $\chi_{\lambda}$ und $\frac{d t_{v}}{d z}$ (R. A. F. \$. 8) nach dem Satze in No. 2., dass die Coefficienten dieser Functionen sich rational aus den Coefficienten von $\varphi(s, z), F(s, z)$ und ihren Ableitungen nach $k$ und aus $(\sigma, k)$ und den $\left(\sigma_{\nu}, \zeta_{\nu}\right)$ zusammensetzen lassen. Ferner sind die Coefficienten der obenerwähnten linearen Gleichungen für die $\gamma, \delta$ rational aus den Coefficienten von $\varphi(s, z), F(s, z)$ und deren Ableitungen nach $k$, ferner aus den Coefficienten von $\chi(s, z), \frac{d t_{\nu}}{d z}, \frac{d U_{\nu}}{d z}$ zusammengesetzt. Hieraus folgt, dass die Grössen $\gamma, \delta$ sich rational aus den Coefficienten von $\varphi(s, z), F(s, z)$ und deren Ableitungen nach $k$, ferner aus den Coefficienten von $\varphi_{\lambda}(s, z)$, und aus $(\sigma, k)$ und den $\left(\sigma_{\nu}, \zeta_{\nu}\right)$ zusammensetzen.

5.

Es sei $K$ der Periodicitätsmodul von $U$ an einem bestimmten Querschnitt, $T_{\nu}, K_{\nu}$ resp. die Periodicitätsmoduln von $t_{v}, U_{v}$ an demselben Querschnitt, so folgt aus Gl. (5.) No. 3:

$$
\frac{\partial^{\lambda} K}{\partial k^{\lambda}}=\delta_{\lambda 1} T_{1}+\delta_{\lambda 2} T_{2}+\cdots+\delta_{\lambda p} T_{p}+\gamma_{\lambda 1} K_{1}+\gamma_{\lambda_{2}} K_{2}+\cdots+\gamma_{\lambda_{p}} K_{p} .
$$

Nehmen wir für $\lambda$ successive $0,1,2, \ldots 2 p$ und eliminiren aus den $2 p+1$ Gleichungen $T_{1}, T_{2}, \ldots T_{p}, K_{1}, K_{2}, \ldots K_{p}$, so erhält man eine Gleichung der Form

$$
\text { (2.) } \boldsymbol{\beta}_{2 p} \frac{\partial^{2 p} \boldsymbol{K}}{\partial k^{2 p}}+\boldsymbol{\beta}_{2 p-1} \frac{\partial^{2 p-1} \boldsymbol{K}}{\partial k^{2 p-1}}+\cdots+\boldsymbol{\beta}_{0} \boldsymbol{K}=\mathbf{0} .
$$

Dieses ist eine lineare Differentialgleichung $2 p^{\text {ter }}$ Ordnung, in welcher $k$ als unabhängige Variable gilt, und welcher die $2 p$ Periodicitätsmoduln von $U$ genügen. Die Coefficienten $\beta$ sind nach voriger No. rationale Functionen der Coefficienten in $\varphi(s, z), F(s, z)$ und deren Ableitungen nach $k$, der Coefficienten von $\varphi_{\lambda}(s, z)$ und der Grössen $(\sigma, k)$ und $\left(\sigma_{\nu}, \zeta_{\nu}\right)$. Allein die Perio- 
330 Fuchs, ïb. gewisse Differentialgleichungen in d. Theorie d. Abelschen Integrale.

dicitätsmoduln von $U$ sind über gewisse Querschnitte erstreckte Integrale, und daher durch die Coefficienten in $\varphi(s, z), F(s, z)$ vollkommen bestimmt, sobald die Querschnitte gegeben sind, und es lassen sich die Verhältnisse der Coefficienten $\beta$ aus diesen Periodicitätsmoduln und ihren Ableitungen ohne Zuhülfenahme anderer Grössen zusammensetzen (s. meine Abh. B. 66 d. J. No. 2). Hieraus folgt, dass die Verhältnisse der Coefficienten $\beta$ nur rationale Functionen der Coefficienten in $\varphi(s, z), F(s, z)$ und deren Ableitungen nach $k$ sind.

Wenn zwischen den Grössen $T_{1}, T_{2}, \ldots T_{p}, K_{1}, K_{2}, \ldots K_{p}$ besondere Beziehungen bestehen, so werden, wenn man für $\lambda$ in Gl. (1.) successive $0,1,2, \ldots q$ setzt, wo $q<2 p$, aus diesen $q+1$ Gleichungen sich schon jene Grössen eliminiren lassen, und es ergiebt sich alsdann eine Differentialgleichung niedrigerer als $2 p^{\text {ter }}$ Ordnung, welcher die sämmtlichen Periodicitätsmoduln von $U$ genügen.

Das in dieser No. entwickelte Theorem ist eine Verallgemeinerung eines auf die hyperelliptischen Integrale bezüglichen in meiner Arbeit B. 71 d. J. No. 8 und 11.

6.

Jacobi hat in diesem Journal B. 36 für das elliptische $\boldsymbol{\vartheta}(0)$ als Function von $\log q=-\pi \frac{K^{\prime}}{K}$ eine Differentialgleichung entwickelt, welche in Bezug auf $\boldsymbol{\vartheta}(\mathbf{0})$ als abhăngige Variable von der dritten Ordnung, in Bezug auf $\boldsymbol{\vartheta}(0)$ und seine Ableitungen nach $\log q$ vom $14^{\text {ten }}$ Grade, und mit rein numerischen Coefficienten behaftet ist.

Fasst man dagegen $\vartheta(0)$ als Function des Moduls $k$ auf, so ist die Differentialgleichung, welcher $\vartheta(0)$ genügt, bei weitem einfacher. Aus der Gleichung

$$
\text { (1.) } \quad \boldsymbol{\theta}(0)=\sqrt{\frac{2 K}{\pi}}
$$

und der bekannten Differentialgleichung

$$
\left(k-k^{3}\right) \frac{d^{2} K}{d k^{2}}+\left(1-3 k^{2}\right) \frac{d K}{d k}-k K=0
$$

oder

$$
\text { (2.) } \frac{d}{d k}\left[k k^{\prime 2} \frac{d K}{d k}\right]-k K=0 \text {, }
$$


Fuchs, üb. gewisse Differentialgleichungen in d. Theorie d. Abelschen Integrale. 331 folgt nämlich, wenn wir mit Jacobi

setzen

$$
\boldsymbol{\vartheta}(\mathbf{0})=\boldsymbol{y}
$$

$$
\frac{d}{d k}\left[k k^{2} \frac{d y^{2}}{d k}\right]-k y^{2}=0 \text {. }
$$

Wenn man wiederum aus dieser Gleichung die Differentialgleichung dritter Ordnung von Jacobi herleiten will, so folgt zunächst aus Gl. (2.), welcher $K$ und $K^{\prime}$ genügen,

also

$$
\frac{d}{d k}\left(\frac{K^{\prime}}{K}\right)=\frac{-\pi}{2 k k^{\prime 2} K^{2}},
$$

Daher ergiebt die Gleichung (3.)

$$
\text { (4.) } \quad \frac{d \log q}{d k}=\frac{2}{k k^{\prime 2} y^{4}} \text {. }
$$

(5.) $8 \cdot \frac{d}{d \log q}\left[\frac{1}{y^{3}} \frac{d y}{d \log q}\right]-k^{2} k^{\prime 2} y^{6}=0$.

Differentiirt man diese Gleichung unter Anwendung der Gl. (4.) nach $\log q$, und eliminirt $k$ aus der entstandenen Gleichung und der Gleichung (5.), so erhält man die Differentialgleichung dritter Ordnung von Jacobi.

Von dieser Differentialgleichung lässt sich, weil sie den Modul $k$ nicht enthält, unmittelbar auf die Theorie der Transformation der elliptischen Functionen Anwendung machen, wie Jacobi l. c. gezeigt hat. Wir wollen nun im Folgenden für $\vartheta(0,0, \ldots 0)$ als Function der Periodicitätsmoduln $a_{i k}$ algebraische Gleichungen zwischen der Function und ihren partiellen Ableitungen nach den $a_{i k}$ herleiten, welche ebenfalls mit rein numerischen Coefficienten behaftet sind. Diese erscheinen als die Verallgemeinerung der Differentialgleichung dritter Ordnung von Jacobi und lassen ebenfalls, da sie von der Wahl des Querschnittssystems unabhängig sind, unmittelbare Anwendung auf die Transformation der Abelschen Functionen zu.

Da jedoch diese Relationen nur unter Voraussetzung der zwischen den Periodicitätsmoduln bestehenden Abhängigkeit gelten, so scheinen uns für manche andere $Z$ wecke, als die sich auf die Transformation beziehenden, solche Relationen den Vorzug zu verdienen, in welchen $\boldsymbol{\vartheta}(0,0, \ldots 0)$ als Function von einander unabhängiger Grössen auftritt. Wir lassen daher die Herleitung von Differentialgleichungen für $\vartheta(0,0, \ldots 0)$ folgen, in welchen die sich nicht aufhebenden Verzweigungspunkte als unabhängige Variable auftreten; diese Differentialgleichungen erscheinen als Verallgemeinerung der Differentialgleichung (3.). 
332 Fuchs, üb. gewisse Differentialgleichungen in d. Theorie d. Abelschen Integrale.

7.

Die Differentialgleichungen der erstgenannten Art, nämlich die zwischen $\boldsymbol{\vartheta}(0,0, \ldots 0)$ und seinen partiellen Ableitungen nach den Periodicitätsmoduln, lassen sich durch rein algebraische Eliminationen erhalten, ohne Kenntniss des Ausdruckes von $\boldsymbol{\vartheta}(0,0, \ldots 0)$ durch die Klassenmoduln oder der Differentialgleichungen, welchen die Periodicitätsmoduln als Functionen derselben Grössen genügen, wenn man von den in meiner vorangehenden Arbeit angestellten $\mathrm{Be}-$ trachtungen Gebrauch macht. Diese Arbeit wollen wir kurz mit $\mathfrak{A}$. citiren.

Es sei $u_{1}, u_{2}, \ldots u_{p}$ ein System linear unabhängiger Integrale erster Gattung, von der Art, dass der Periodicitätsmodul von $u_{\mathfrak{a}}$ am Querschnitte $a_{\mathfrak{a}}$ gleich $\pi i$, an allen übrigen $a$ gleich Null, am Querschnitte $b_{v}$ gleich $a_{\mathfrak{a} v}$ (und $a_{\mathfrak{a} v}=a_{\nu \mathfrak{a}}$ ), (R. A. F. $9.18-21$ ), so ist bekanntlich

eine rationale Function von $(s, z)$.

$$
\boldsymbol{E}_{\lambda \mu}(s, \mathfrak{z})=\frac{\partial^{2} \log \boldsymbol{\vartheta}\left(\underset{\mathfrak{a}}{p}\left(u_{\mathfrak{\alpha}}\right)\right)}{\partial u_{\lambda} \partial u_{\mu}}
$$

Ist $\mu$ ein beliebiger Punkt der Fläche $T$, und $\alpha_{1}^{(())}, \alpha_{2}^{(0)}, \ldots \alpha_{p}^{(1)}$ das mit $\mu$ durch die Gleichung

$$
\psi_{\mu}(s, z)=0
$$

verknüpfte Hauptsystem (s. m. $\mathfrak{A}$. No.1-6), und disponirt man über die in den $u_{\mathfrak{a}}$ enthaltenen Constanten derart, dass man setzt:

$$
\text { (1.) } \quad u_{\mathfrak{a}}=\int_{\mu}^{\xi} d u_{\mathfrak{a}}, \quad \mathfrak{a}=1,2, \ldots p,
$$

wo $\xi$ der einem beliebigen Werthenpaare $(s, z)$ entsprechende Punkt der Fläche $T$ ist, so wird $\vartheta\left(\begin{array}{l}p \\ \mathfrak{a}\end{array}\left(u_{\mathfrak{a}}\right)\right)$ in den Punkten $\alpha_{1}^{(())}, \alpha_{2}^{(())}, \ldots \alpha_{p}^{(1)}$ unendlich klein erster Ordnung (s. m. $\mathfrak{A}$. No. 3 Gl. (2.)), also $E_{\lambda \mu}(s, z)$ in denselben Punkten unendlich gross zweiter Ordnung, sonst aber überall endlich. Die Function $\psi_{\mu}(s, z)$ wird ebenfalls in diesen Punkten unendlich klein zweiter Ordnung (s. m. '- . No. 2), also ist

$$
G_{\lambda \mu}(s, z)=E_{\lambda \mu}(s, z) \psi_{\mu}(s, z)
$$

eine ganze rationale Function von $(s, z)$. Da $u_{\mathfrak{a}}$ für jeden Punkt der Fläche $T$ endlich ist, so ist für $z=\infty$ oder $s=\infty G_{\lambda \mu}(s, z)$ ebenso oft unendlich wie $\psi_{\mu}$, demnach ist $G_{\mu}(s, z)$ wie $\psi_{\mu}$ in Bezug auf $s$ vom Grade $n-1$, in Bezug auf $z$ vom Grade $m-1$, also

(3.) $G_{\lambda \mu}(s, z)=G_{0}+G_{1} s+G_{2} s^{2}+\cdots+G_{n-1} s^{n-1}$,

wo $G_{0}, G_{1}, \ldots G_{n-1}$ ganze rationale Functionen $m-1^{\text {ten }}$ Grades von $z$ sind. 
Fuchs, üb. gewisse Differentialgleichungen in d. Theorie d. Abelschen Integrale. 333

Wir setzen zur Abkürzung

$$
\text { (4.) }\left[\frac{\partial^{q} \log \vartheta\left(\underset{\mathfrak{a}}{p}\left(u_{\mathfrak{a}}\right)\right)}{\partial u_{\mathfrak{a}} \partial u_{\mathfrak{b}} \partial u_{\mathfrak{c}} \ldots}\right]_{\xi=\mu}=L_{\mathfrak{a b c} \ldots,}
$$

wo $q$ die Anzahl der $u$, nach denen differentiirt wird, bedeutet. Differentiirt man nun die Gleichung (3.), indem man die Identität:

$$
\text { (5.) } \quad \frac{d E_{\lambda \mu}(s, \mathfrak{z})}{d z}=\sum_{\mathfrak{l}}^{p} \frac{\partial^{3} \log \vartheta\left(\begin{array}{l}
p \\
\mathfrak{a}
\end{array}\left(u_{\mathfrak{a}}\right)\right)}{\partial u_{\lambda} \partial u_{\mu} \partial u_{\mathfrak{b}}} \frac{d u_{\mathfrak{b}}}{d z}
$$

anwendet, $m n$ mal, und setzt $\xi=\mu$, so bilden diese Gleichungen mit Gl. (3.) (ebenfalls für $\xi=\mu$ ) zusammen $m n+1$ Gleichungen, aus welchen die $m n$ Constanten der Functionen $G_{0}, G_{1}, \ldots G_{n-1}$ eliminirt werden können. Das

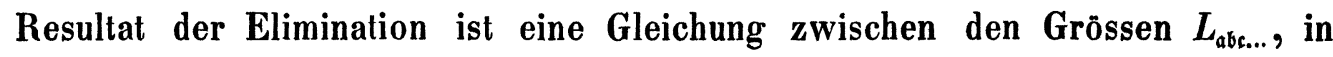
welchen die Ordnung der Differentiation höchstens gleich $m n+2$, und zwischen den Coefficienten von $\frac{d u_{\mathfrak{a}}}{d z}$ und $\psi_{\mu}$ und der Grösse $\mu$. Bezeichnen wir diese Gleichung mit

$$
(\boldsymbol{A})=\mathbf{0} \text {. }
$$

Bildet man die ferneren Ableitungen der Gleichung (3.) bis zur $m n+l^{\text {ten }}$ Ordnung, setzt $\xi=\mu$ und eliminirt aus jeder derselben mit Hülfe von $m n$ der ersten Gleichungen die Constanten in $G_{0}, G_{1}, \ldots G_{n-1}$, so mögen die neuentstandenen Gleichungen mit

$$
\left(A^{\prime}\right)=0, \quad\left(A^{\prime \prime}\right)=0, \quad \cdots \quad\left(A^{(l)}\right)=0
$$

bezeichnet werden. Dieselben werden theilweise identisch erfüllt, theilweise von einander abhängig sein können. Immer aber wird für ein hinlänglich grosses $l$ eine genügende Anzahl von Gleichungen vorhanden sein, um mit Hülfe der Gleichungen, welche zwischen den $\alpha$ und $\mu$ bestehen (s. m. $\mathfrak{A}$. No. 5), und der Gl. (1.) No. 1 die Grösse $\mu$, die Coefficienten von $F(s, z)$, $\frac{d u_{\mathfrak{a}}}{d z}$ und von $\psi_{\mu}$ zu eliminiren. Das Resultat ist eine Gleichung zwischen den Grössen $L_{a b c . . .}$, in welchen die Ordnung der Differentiation höchstens gleich $m n+l+2$, mit rein numerischen Coefficienten.

Solcher Gleichungen erhält man $\frac{p(p+1)}{2}$, wenn man für $\lambda, \mu$ alle zulässigen Combinationen macht. 
334 Fuchs, üb. gewisse Differentialgleichungen in d. Theorie d. Abelschen Integrale.

8.

Da $\vartheta\left(\mathfrak{a}_{1}^{p}\left(u_{\mathfrak{a}}\right)\right)$ und jede ihrer Ableitungen gerader Ordnung eine gerade Function der $u$, jede Ableitung ungerader Ordnung eine ungerade Function derselben Grössen ist, so verschwinden die letzteren, wenn in denselben $u_{1}=u_{2}=\cdots=u_{p}=0$ gesetzt wird. Daher verschwinden auch die Ableitungen ungerader Ordnung von $\log \vartheta\left(\mathfrak{a}\left(u_{\mathfrak{a}}\right)\right)$ für dieselben Werthe.

Die bekannten Relationen

$$
\text { (1.) } \frac{4 \partial \vartheta}{\partial a_{\mu \mu}}=\frac{\partial^{2} \vartheta}{\partial u_{\mu}^{2}}, \frac{2 \partial \vartheta}{\partial a_{\mu \mu^{\prime}}}=\frac{\partial^{2} \vartheta}{\partial u_{\mu} \partial u_{\mu^{\prime}}}
$$

lassen sich folgendermassen schreiben:

$$
\text { (2.) }\left\{\begin{array}{l}
\frac{4 \partial \log \vartheta}{\partial a_{\mu \mu}}=\frac{\partial^{2} \log \vartheta}{\partial u_{\mu}^{2}}+\left(\frac{\partial \log \vartheta}{\partial u_{\mu}}\right)^{2} \\
\frac{2 \partial \log \vartheta}{\partial a_{\mu \mu^{\prime}}}=\frac{\partial^{2} \log \vartheta}{\partial u_{\mu} \partial u_{\mu^{\prime}}}+\frac{\partial \log \vartheta}{\partial u_{\mu}} \frac{\partial \log \vartheta}{\partial u_{\mu^{\prime}}} .
\end{array}\right.
$$

Für $u_{1}=u_{2}=\cdots=u_{p}=0$ gehen die Relationen (2.) über in

$$
\text { (3.) }\left\{\begin{array}{l}
\frac{4 \partial \log \vartheta(0,0, \ldots 0)}{\partial a_{\mu \mu}}=\left[\frac{\partial^{2} \log \vartheta}{\partial u_{\mu}^{2}}\right]_{u=0}, \\
\frac{2 \partial \log \vartheta(0,0, \ldots 0)}{\partial a_{\mu \mu^{\prime}}}=\left[\frac{\partial^{2} \log \vartheta}{\partial u_{\mu} \partial u_{\mu^{\prime}}}\right]_{u=10},
\end{array}\right.
$$

Sind $\mathfrak{a}, \mathfrak{b}, \mathfrak{c}, \mathfrak{b}, \ldots$ unter einander verschieden, so folgt aus der Form der Thetafunction, als Verallgemeinerung von (1.),

$$
\text { (4.) } \frac{2^{\lambda} \partial^{\lambda} \vartheta}{\partial a_{\mathfrak{a} \mathfrak{b}} \partial a_{\mathfrak{c b}} \cdots}=\frac{\partial^{2 \lambda} \vartheta}{\partial u_{\mathfrak{a}} \partial u_{\mathfrak{b}} \partial u_{\mathfrak{l}} \partial u_{\mathfrak{b}} \ldots}
$$

wo $2 \lambda$ die Anzahl der $u$, nach welchen auf der rechten Seite differentiirt wird, bedeutet. Sind irgend welche unter den Grössen $\mathfrak{a}, \mathfrak{b}, \mathfrak{c}, \mathfrak{b}, \ldots$ einander gleich, so wird der Exponent von 2 auf der linken Seite der Gleichung (4.) um eine leicht bestimmbare Zahl grösser.

Aus dieser Gleichung folgt, dass man die partiellen Ableitungen gerader Ordnung von $\log \vartheta$ für $u_{1}=u_{2}=\cdots=u_{p}=0$ als rationale Functionen mit numerischen Coefficienten der Ableitungen von $\vartheta(0,0, \ldots 0)$ nach den $a_{i k}$ oder auch als ganze rationale Functionen der Ableitungen von $\log \vartheta(0,0, \ldots 0)$ nach den $a_{i k}$ darstellen kann.

Mit Hülfe dieser Relationen führen wir die zwischen den Grössen $L_{\mathfrak{a b} \ldots . .}$ in voriger No. gefundenen Beziehungen in Gleichungen zwischen den 
Ableitungen von $\log \vartheta(0,0, \ldots 0)$ nach den Periodicitätsmoduln $a_{i k}$ über, wie sie oben angekündigt worden.

Da jedes eigentliche System $(\alpha)$, welches mit $\mu$ durch eine Gleichung $\psi_{\mu}=0$ verknüpft ist, als Hauptsystem gelten kann, wenn man das Querschnittssystem $a, b$ zweckmässig wählt (s. m. $\mathfrak{A}$. No. 5), so unterliegt das in voriger und dieser No. beschriebene Verfahren nicht der Schwierigkeit, welche mit dem Aussondern des Hauptsystems verbunden ist (s. m. $\mathfrak{A}$. No. 5 und 6). Wählt man vielmehr ein beliebiges eigentliches System $(\alpha)$ als Hauptsystem und bildet mit dem zugehörigen $\psi_{\mu}, \mathrm{d}$. h. demjenigen $\psi_{\mu}$, durch welches das System $(\alpha)$ mit $\mu$ verknüpft ist, die Gleichung (2.) vor. No., so erhält man die oben entwickelten Relationen unter Voraussetzung der entsprechenden Querschnittszerlegung.

Da jedoch die den verschiedenen eigentlichen Systemen $(\alpha)$ zugehörigen $\psi_{\mu}$ sich nur durch die in den Coefficienten derselben auftretenden Grössensysteme $(\alpha)$ unterscheiden, die letzteren aber bei der oben angedeuteten Elimination herausfallen, so folgt: Die gefundenen Relationen bleiben ungeändert für jede beliebige Art der Querschnittszerlegung.

9.

Um für die elliptischen Functionen auf diesem Wege die Rechnung durchzuführen, sei

$$
\text { (1.) } s^{2}=R(z)
$$

wo

$$
R(z)=\left(1-z^{2}\right)\left(1-k^{2} z^{2}\right),
$$

ferner $A$ der Periodicitätsmodul von $\int \frac{d z}{s}$ am Querschnitte $a$, und es werde gesetzt

$$
\text { (2.) } \quad u=\frac{i \pi}{A} \int_{\mu}^{\xi} \frac{d z}{s},
$$

so hat $u$ am Querschnitte $a$ den Periodicitätsmodul $\pi i$, am Querschnitte $b$ heisse er $\tau$. Es bleibt hierbei unentschieden, wie die beiden Querschnitte $a$ und $b$ zu legen sind.

Soll $\vartheta\left(\int_{\mu}^{\xi} d u-\int_{\alpha}^{\eta} d u+P\right)$, wo $P$ einen der Werthe

$$
\frac{\pi i}{2}, \frac{\tau}{2}, \frac{\pi i}{2}+\frac{\tau}{2}
$$


336 Fuchs, üb. gewisse Differentialgleichungen in d. Theorie d. Abelschen Integrale.

bedeutet, verschwinden für $\xi=\eta$, wo $\eta$ ein beliebiger Punkt der Fläche $T$ ist, so ist bekanntlich

also

$$
\text { (3.) } \int_{\mu}^{\alpha} d u+P \equiv \frac{\pi i}{2}+\frac{\tau}{2}
$$

$$
\text { (4.) } 2 \int_{\mu}^{\alpha} d u \equiv 0 \text {. }
$$

Die den drei verschiedenen Systemen halber Periodicitätsmoduln $\boldsymbol{P}$ nach Gl. (3.) zugehörigen Werthe $\alpha$, oder die Functionen $\psi_{\mu}$, durch welche sie mit $\mu$ verknüpft sind, können also, wie aus der Gleichung (4.) folgt, nach dem Abelschen Theorem in diesem Falle folgendermassen gefunden werden:

Es sei, $\left(s_{\mu}, \mu\right)$ das dem Punkte $\mu$ zugehörige Werthsystem $(s, z)$, so setzen wir

$$
\text { (5.) } \quad \psi_{\mu}(s, z)=a_{0}+a_{1} z+a_{2} z^{2}+c s
$$

und bestimmen die Constanten $a$ und $c$ durch folgende Bedingungsgleichungen:

$$
\text { (6.) } \quad \psi_{\mu}\left(-s_{\mu}, \mu\right)=0, \quad \psi_{\mu}^{\prime}\left(-s_{\mu}, \mu\right)=0,
$$

wo $\psi_{\mu}^{\prime}(s, z)$ die totale Ableitung von $\psi_{\mu}(s, z)$ nach $z$ bedeutet.

Die Bedingungen, dass $\psi_{\mu}(s, z)$ in $\alpha$ unendlich klein zweiter Ordnung wird, sind, wenn $\left(s_{\alpha}, \alpha\right)$ das dem Punkte $\alpha$ zugehörige Werthsystem $(s, z)$ ist,

(7.) $\quad \psi\left(s_{\alpha}, \alpha\right)=0, \quad \psi^{\prime}\left(s_{\alpha}, \alpha\right)=0$.

Die Elimination von $a_{0}, a_{1}, a_{2}, c$ aus den Gleichungen (6.) und (7.) ergiebt für $\alpha$ folgende Gleichung:

$$
\text { (8.) }(\alpha-\mu)\left[2\left(s_{\mu}+s_{\alpha}\right)+(\alpha-\mu)\left(s_{\mu}^{\prime}-s_{\alpha}^{\prime}\right)\right]=0,
$$

wo $s^{\prime}$ wieder die Ableitung von $s$ nach $z$ bedeutet. Die Gleichungen (6.) und (7.) ergeben alsdann die Verhältnisse der Coefficienten von $\psi_{\mu}(s, z)$.

Unter Voraussetzung irgend einer Lage der Querschnitte $a$ und $b$, sei $\alpha$ diejenige Wurzel der Gleichung (8.), für welche $\vartheta\left(\int_{\mu}^{\xi} d u\right)$ verschwindet, also das Hauptsystem des allgemeinen Falles, welches sich hier auf ein Glied reducirt, so ist in diesem Falle

$$
\text { (9.) } E(s, z)=\frac{d^{2} \log \vartheta(u)}{d u^{2}}
$$

und

$$
\text { (10.) } \quad G(s, z)=G_{0}+G_{1} s,
$$

wo $G_{0}$ vom zweiten, $G_{1}$ von nullten Grade ist. 
Bildet man die Ableitungen der Gleichung (10.) nach $z$ bis zur zehnten Ordnung, und setzt $\xi=\mu$, so kann man aus den ersten 4 der 11 Gleichungen die Coefficienten von $G_{0}$ und $G_{1}$ bestimmen, diese in die nächstfolgenden substituirt liefern 4 von einander unabhängige Gleichungen, aus welchen man mit Hinzuziehung von Gleichung (8.) $k, \frac{i \pi}{A}, \mu$ eliminiren kann. Das Eliminationsresultat ist eine Gleichung zwischen $L_{4}, L_{6}, L_{8}, L_{10}$, wenn man setzt:

$$
\left[\frac{d^{\mathfrak{a}} \log \vartheta(u)}{d u^{\mathfrak{a}}}\right]_{\mathfrak{a}=0}=L_{\mathfrak{\alpha}} .
$$

Nach der vorigen No. lassen sich diese Grössen durch die Ableitungen von $\log \boldsymbol{\vartheta}(0)$ nach $\tau$ darstellen. Die Differentialgleichung für $\boldsymbol{\vartheta}(0)$, welche man erhält, ist fünfter Ordnung. Sie ergiebt sich aus der Jacobischen, für $\tau=\log q$, durch zweimalige Differentiation nach $\tau$.

10.

Um die Differentialgleichung herzuleiten, welcher $\vartheta(0,0, \ldots 0)$ als Function eines der sich nicht aufhebenden Verzweigungspunkte genügt, wenden wir den Ausdruck von $\frac{\partial \log \vartheta(0,0, \ldots 0)}{\partial k}$ als Function von $k$ an.

Es bedeute nämlich $U_{1}, U_{2}, \ldots U_{p}$ wie in No. 3 ein System linearunabhängiger Integrale erster Gattung, und $A_{\mathfrak{a}}^{(\mu)}$ den Periodicitätsmodul von $U_{\mathfrak{a}}$ am Querschnitte $a_{\mu}$; setzt man ferner die Determinante

$$
\left|\begin{array}{cccc}
A_{1}^{(1)}, & A_{2}^{(1)}, & \ldots & A_{p}^{(1)} \\
A_{1}^{(2)}, & A_{2}^{(2)}, & \ldots & A_{p}^{(2)} \\
\vdots & \vdots & & \vdots \\
A_{1}^{(p)}, & A_{2}^{(p)}, & \ldots & A_{p}^{(p)}
\end{array}\right|=\nabla
$$

so ist (s. m. . No. 8 Gl. (8.) und No. 9 Gl. (4.))

$$
\text { (1.) } \frac{\partial \log \boldsymbol{\vartheta}(0,0, \ldots 0)}{\partial k^{*}}=\frac{1}{2} \frac{\partial \log \nabla}{\partial k}+\boldsymbol{R} \text {, }
$$

wo $R$ auf algebraische Weise sowohl explicite von $k$ als auch von den Coefficienten von $\varphi_{\mathfrak{a}}(s, z)$ und $F(s, z)$ abhängt.

Nach No. 5 Gleichung (1.) ist:

(2.) $\frac{\partial^{\lambda} A_{a}^{(\mu)}}{\partial k^{\lambda}}=\delta_{\lambda 1}^{(\mathfrak{a})} T_{1}^{(\mu)}+\delta_{\lambda 2}^{(\mathfrak{a})} T_{2}^{(\mu)}+\cdots+\delta_{\lambda p}^{(\mathfrak{a})} T_{p}^{(\mu)}+\gamma_{\lambda 1}^{(\mathfrak{a})} A_{1}^{(\mu)}+\gamma_{\lambda 2}^{(\mathfrak{a})} A_{2}^{(\mu)}+\cdots+\gamma_{\lambda p}^{(\mathfrak{a})} A_{p}^{(\mu)}$, wo $T_{\mathfrak{a}}^{(\mu)}$ den Periodicitätsmodul von $t_{\mathfrak{a}}$ am Querschnitte $a_{\mu}$ bedeutet, und $\gamma$, $\delta$ sich rational aus den Coefficienten von $\varphi_{\mathfrak{a}}(s, z), F(s, z)$ und deren Ableitungen nach $k$, und aus $(\sigma, k)$ und den $\left(\sigma_{\nu}, \zeta_{\nu}\right)$ zusammensetzen lassen. 
338 Fuchs, üb. gewisse Differentialgleichungen in d. Theorie d. Abelschen Integrale.

Differentiiren wir die Gleichung (1.) $2 p^{2}$ mal nach $k$, und setzen für die Ableitungen von $A_{\mathfrak{a}}^{(\mu)}$ ihre Werthe aus (2.), so kann man aus den entstandenen $2 p^{2}+1$ Gleichungen die $2 p^{2}$ Grössen $A_{\mathfrak{a}}^{(\mu)}, T_{\mathfrak{a}}^{(\mu)}$ eliminiren. Das Resultat der Elimination ist eine algebraische Gleichung zwischen den Ableitungen von $\vartheta(0,0, \ldots 0)$ als Function von $k$, deren Coefficienten algebraisch aus den Coefficienten von $\varphi_{\mathfrak{a}}(s, z), F(s, z)$ und deren Ableitungen nach $k$, und aus $(\sigma, k)$ und den $\left(\sigma_{\nu}, \zeta_{\nu}\right)$ zusammengesetzt sind.

Differentiirt man diese Gleichung $p$ mal, so kann man aus den entstandenen $p+1$ Gleichungen, unter Zuhülfenahme der Gleichungen $\boldsymbol{F}\left(\sigma_{\nu}, \zeta_{\nu}\right)=0$, die $p$ Grössenpaare $\left(\sigma_{\nu}, \zeta_{\nu}\right)$ eliminiren, und erhält schliesslich als die gesuchte Verallgemeinerung der Differentialgleichung (3.) in No. 6. eine Differentialgleichung

$$
\text { (3.) } \quad M_{k}=0 \text {, }
$$

deren Coefficienten sich algebraisch aus den Coefficienten von $\varphi_{\mathfrak{a}}(s, z), F(s, z)$ und deren Ableitungen nach $k$ und aus $(\sigma, k)$ zusammensetzen.

11.

Unter Voraussetzung der in No. 3-5 entwickelten Formeln und der Differentialgleichung (3.) vor. No. lassen sich die in No. 7 u. 8 behandelten Relationen zwischen den Ableitungen von $\log \vartheta(0,0, \ldots 0)$ nach den Periodicitätsmoduln $a_{i k}$ auch auf folgende Weise herleiten.

Bekanntlich ist

$$
\text { (1.) } \quad u_{\mathfrak{a}}=\frac{i \pi}{\nabla} \sum_{\mathfrak{1}}^{p} \frac{\partial \nabla}{\partial A_{\mathfrak{b}}^{(\mathfrak{a})}} U_{\mathfrak{b}}, \quad \mathfrak{a}=1,2, \ldots p
$$

ein System linearunabhängiger Integrale erster Gattung von der Beschaffenheit, wie es in No. 7 gefordert wird.

Es werde vorausgesetzt, dass die Coefficienten von $\varphi_{\mathfrak{a}}(\stackrel{n-2}{s,}, z)$, als Functionen eines jeden der sich nicht aufhebenden Verzweigungspunkte, algebraischen Differentialgleichungen genügen, also beispielsweise, wie die Coefficienten von $F(s, z)$, algebraische Functionen von $k$ sind. Da die Grössen $A_{\mathfrak{a}}^{(\mu)}$ nach No. 5 linearen Differentialgleichungen genügen, deren Coefficienten rationale Functionen der Coefficienten von $\varphi_{\mathfrak{a}}(s, z), F(s, z)$ und deren $\mathrm{Ab}-$ leitungen nach $k$ vorstellen, so sind auch die Coefficienten von $\frac{d u_{\mathfrak{a}}}{d z}$ Functionen von $k$, welche algebraischen Differentialgleichungen genügen. - Unter derselben Voraussetzung ergiebt sich, dass die Coefficienten der Gleichung (3.) vor. No. als Functionen von $k$ algebraischen Differentialgleichungen genügen. 
Fuchs, üb. gewisse Differentialgleichungen in d. Theorie d. Abelschen Integrale. 339

Nun ist nach No. 5 Gl. (1.):

(2.) $\quad \frac{\partial^{\lambda} a_{\alpha \mu}}{\partial k^{\lambda}}=\delta_{\lambda 1}^{(\mathfrak{a})} T_{1}^{(\mu)}+\delta_{\lambda 2}^{(\mathfrak{\alpha})} T_{2}^{(\mu)}+\cdots+\delta_{\lambda p}^{(\mathfrak{\alpha})} T_{p}^{(\mu)}+\gamma_{\lambda 1}^{(\mathfrak{a})} a_{1 \mu}+\gamma_{\lambda 2}^{(\mathrm{a})} a_{2 \mu}+\cdots+\gamma_{\lambda p}^{(\mathfrak{a})} a_{p \mu}$,

wo die Grössen $\gamma, \delta$ sich nach No. 4 rational aus den Coefficienten der Functionen $F(s, z), \frac{d u_{\mathfrak{a}}}{d z}$ und ihrer Ableitungen nach $k$, und aus den Grössen $(\sigma, k)$ und den $\left(\sigma_{\nu}, \zeta_{\nu}\right)$ zusammensetzen.

Es ist aber:

$$
\text { (3.) } \quad \frac{\partial \log \vartheta(0,0, \ldots 0)}{\partial k}=\sum_{\mathfrak{a} \mathfrak{b}} \frac{\partial \log \vartheta(0,0, \ldots 0)}{\partial a_{\mathfrak{a} \mathfrak{b}}} \frac{\partial a_{\mathfrak{a b}}}{\partial k} \text {. }
$$

Differentiiren wir diese Gleichung bis zur $l^{\text {ten }}$ Ordnung nach $k$, indem wir stets $\boldsymbol{9}(0,0, \ldots 0)$ als Function der $a_{\mu \nu}$ und diese wieder als Functionen von $k$ behandeln, und setzen in jeder dieser Gleichungen für die Ableitungen von $a_{\mu \nu}$ nach $k$ deren Werthe aus Gl. (2.), so können wir $l$ so gross wählen, dass wir aus den entstehenden $l+1$ Gleichungen, unter Zuhülfenahme der successiven Ableitungen der Gleichung (3.) vor. No. die Grössen $(\sigma, k),\left(\sigma_{\nu}, \zeta_{\nu}\right)$, $T_{\mathfrak{a}}^{(\mu)}, a_{\mu \nu}$ und die. Ableitungen von $\vartheta(0,0, \ldots 0)$ nach $k$ eliminiren können. Die Coefficienten der resultirenden Gleichung zwischen den Ableitungen von $\vartheta(0,0, \ldots 0)$ nach den $a_{\mu \nu}$

$$
\text { (4.) }\left(\Lambda_{k}\right)=0
$$

hängen auf algebraische Weise ab von den Coefficienten von $F(s, z), \frac{d u_{\mathfrak{a}}}{d z}$ und ihren Ableitungen nach $k$, ferner von den Coefficienten der Gl. (3.) vor. No. und ihren Ableitungen nach $k$. Mit Hülfe der successiven Ableitungen der einzelnen algebraischen Differentialgleichungen, welchen diese verschiedenen Grössen genügen, lassen sie sich alle aus der Gl. (4.) und deren Ableitungen eliminiren, und man erhält eine Gleichung

$$
\text { (5.) }\left(\boldsymbol{\Lambda}_{k}^{\prime}\right)=\mathbf{0} \text {, }
$$

welche eine Relation zwischen den Ableitungen von $\vartheta(0,0, \ldots 0)$ nach den $a_{\mu \nu}$ ausdrückt, mit rein numerischen Coefficienten.

Den verschiedenen sich nicht aufhebenden Verzweigungspunkten $k$ entsprechend ergiebt sich eine gewisse Anzahl von Relationen dieser Art.

- Greifswald im März 1871. 